\title{
Theoretical and numerical analysis of a heat pump model utilizing Dufour effect
}

\author{
Minoru Hoshina and Koji Okuda \\ Division of Physics, Hokkaido University, Sapporo 060-0810, Japan \\ Received: date / Revised version: date
}

\begin{abstract}
A heat pump model utilizing the Dufour effect is proposed, and studied by numerical and theoretical analysis. Numerically, we perform MD simulations of this system and measure the cooling power and the coefficient of performance (COP) as figures of merit. Theoretically, we calculate the cooling power and the COP from the phenomenological equations describing this system by using the linear irreversible thermodynamics and compare the theoretical results with the MD results.
\end{abstract}

PACS. XX.XX.XX No PACS code given

\section{Introduction}

The Dufour effect [1] induces the temperature difference from the mole fraction difference in the mixture fluid system as the Peltier effect [2] does from the electric potential difference. Although the Peltier effect is widely applied to various heat pumps [3, 4, it has not been studied whether an application of the Dufour effect to heat pumps is possible or not. In this paper, we propose a heat pump model utilizing the Dufour effect and study this model by numerical and theoretical analysis.

The Dufour effect is well studied by the experiments [1,5, 6, 7, 8, 9, 10, 11] and theoretical approaches such as the linear irreversible thermodynamics [12,13, the ChapmanEnskog theory [14,15, the phenomenology [16, and other methods 17, 18. In 1873, L. Dufour mixed the two gases of different molecular-weights and discovered a temperature fall in the higher-molecular-weight gas during the diffusive mixing process [1. The theory describing this effect was first developed by Chapman and Enskog by applying the kinetic theory to the microscopic analysis of the binary gas mixture 14, in which the temperature $T$ and the numberdensities of molecules $n_{A}$ and $n_{B}$ of the two components $A$ and $B$ are non-uniform in space. They derived that the heat current $\boldsymbol{J}_{Q}$ can be written as

$$
\boldsymbol{J}_{Q}=-\kappa \nabla T-n k_{B} T^{2} D^{\prime \prime} \nabla x_{A},
$$

where $D^{\prime \prime}$ is the Dufour coefficient, $\kappa$ is the thermal conduction coefficient, $k_{B}$ is the Boltzmann constant, $n$ is the total number-density of all the components, i.e. $n=$ $n_{A}+n_{B}$, and $x_{A}$ is the mole fraction of the component $A$ defined as $x_{A} \equiv n_{A} / n$. The result $D^{\prime \prime}<0$ can also be derived from their theory when the molecular mass of the component $A$ is lower than that of the component $B$ (i.e. $m_{A}<m_{B}$ ) in some special cases of the intermolecular potential. This result implies that the heat current tends to flow from $A$-rich part to $B$-rich part, which is consistent with the above experiment by Dufour.

The organization of this paper is as follows. We construct a heat pump model utilizing the Dufour effect in Section 2, and the usefulness of this model as a heat pump is confirmed numerically using the molecular dynamics (MD) simulation 19 in Section 3 Next, by using the linear irreversible thermodynamics [12, we theoretically analyze this model in a simple case where the heat pump is driven very slowly and attached to the two heat baths whose temperature difference is zero or small, and compare the theoretical results with the data obtained numerically by the MD simulation in Section 4 . Finally, we summarize this study in Section 5 .

\section{Model}

The main idea of our model is the following. Since the Dufour effect occurs only during the transient diffusive mixing process, as far as we know from the previous experimental studies $[1,5,6,7,8,9,10,11$, it is difficult to keep the Dufour effect constant like the steady state of the Peltier effect. For this reason, we need a process that separates the components of the mixture, besides the diffusive mixing process. In our model, an external electric field is used for the separation of the mixture.

Consider a gas mixture of the two components $A$ and $B$, and assume that the molecular mass of the component $A$ is lower than that of $B$, so that $m_{A}<m_{B}$. To separate the mixed components into $A$ and $B$ by an electric field, electric charges $q_{A}$ and $q_{B}$ are given to each molecule of $A$ and $B$, respectively, and we assume $q_{A}=-q$ and $q_{B}=q(q>0)$ for simplicity. Though the molecules have electric charge, Coulomb interaction between them is ig- 


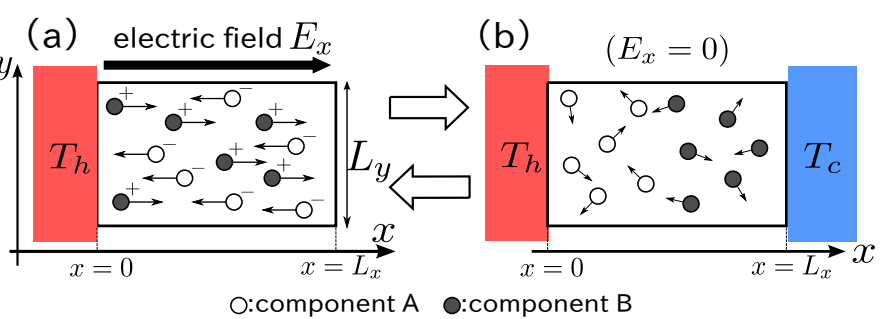

Fig. 1. Schematic illustration of the system and the processes of the heat pump model. (a) In the separating process, only the heat bath with $T_{h}$ is attached and the electric field $E_{x}$ is applied. (b) In the mixing process, both of the heat baths with $T_{h}$ and $T_{c}$ are attached and the electric field is turned off.

nored throughout the paper 1 . The particle numbers of the components in the system are $N_{A}$ and $N_{B}$, and other properties of the components $A$ and $B$ such as the particle interaction or the shape of the molecules are assumed to be identical.

This gas mixture is contained in the system as schematically depicted in Figure 1 The system is a two-dimensional rectangle with the size $L_{x} \times L_{y}$. To pump a heat from the heat bath with a low temperature $T_{c}$ to the heat bath with a high temperature $T_{h}$, two procedures, (a) separating process and (b) mixing process are alternately repeated. The details of these processes depicted in Figure 1 are described as follows.

(a) Separating process : during this process, the heat bath with $T_{h}$ is attached to the boundary at $x=0$, and the insulated wall is placed on the boundary at $x=L_{x}$. Furthermore, a static external electric field $E_{x}=E(>$ $0)$ is applied in the $x$-direction. After continuing this process for a duration $\Delta t_{\mathrm{sep}}$, the system is switched to the mixing process.

(b) Mixing process : during this process, the heat baths with $T_{h}$ and $T_{c}\left(T_{h}>T_{c}\right)$ are attached to the boundary at $x=0$ and $x=L_{x}$, respectively, and the electric field is turned off $\left(E_{x}=0\right)$. After continuing this process for a duration $\Delta t_{\text {mix }}$, the system is switched to the separating process.

In the separating process, the components of the gas mixture are separated by the external field $E_{x}$ so that a negative gradient of the mole fraction $\partial x_{A} / \partial x<0$ is established. The heat energy due to the work done by the external field $E_{x}$ leaks into the heat bath with the temperature $T_{h}$, and the system approaches the equilibrium state of the total system at the temperature $T_{h}$ if the duration $\Delta t_{\text {sep }}$ is taken sufficiently long. In the mixing process, a diffusive mixing of the components $A$ and $B$ occurs. As seen from equation (11) and $m_{A}<m_{B}$ (therefore $D^{\prime \prime}<0$ ), a heat current flows in the negative $x$-direction due to the

\footnotetext{
${ }^{1}$ We note that our purpose in this paper is to suggest the possibility of the heat pump utilizing the Dufour effect. Though we use the electric field and charged particles to separate the components clearly, we consider that this method can be replaced with another such method as using gravity to realize this heat pump. This is discussed as a remaining problem in the last paragraph of Section 5
}

Dufour effect so that an amount of heat is expected to be pumped from the heat bath with $T_{c}$ to the heat bath with $T_{h}$.

\section{MD simulation of the model}

\subsection{The simulation model}

In our simulation model, the time evolution of the system is governed by a Hamiltonian

$$
\begin{array}{r}
\mathcal{H}=\sum_{i=1}^{N} \frac{\boldsymbol{p}_{i}^{2}}{2 m_{i}}+\sum_{i<j} U^{\mathrm{int}}\left(\left|\boldsymbol{r}_{i}-\boldsymbol{r}_{j}\right|\right)-\sum_{i=1}^{N} q_{i} E_{x}(t) \tilde{x}_{i} \\
\left(N \equiv N_{A}+N_{B}\right),
\end{array}
$$

where $\boldsymbol{p}_{i}, \boldsymbol{r}_{i}, m_{i}, q_{i}$, and $\tilde{x}_{i}$ denote the momentum, position, mass, electric charge, and $x$-coordinate of the $i$ th particle, respectively. $U^{\text {int }}$ denoting the interaction potential for the center-to-center distance $r$ of the particles is taken to be a hard Herzian potential [20,21, 22,

$$
U^{\text {int }}(r)= \begin{cases}Y|\sigma-r|^{\frac{5}{2}} & (r \leq \sigma) \\ 0 & (\sigma<r)\end{cases}
$$

where $\sigma$ is the diameter of the particle, and a constant $Y$ is taken to be $Y=10^{5} \epsilon \sigma^{-\frac{5}{2}}$ with an energy unit $\epsilon$. The external electric field $E_{x}(t)$ is defined as

$$
E_{x}(t)= \begin{cases}E & \text { (in the separating process) } \\ 0 & \text { (in the mixing process) }\end{cases}
$$

where $E$ is a positive constant. Note that the electric charge of particles is used only to separate the components and for simplicity Coulomb interaction between them is ignored in our simulations.

The periodic boundary condition is imposed in the $y$ direction. The boundary of the $x$-direction at $x=L_{x}$ is the elastically reflecting wall in the separating process, and the thermalizing wall 23 . with the temperature $T_{c}$ in the mixing process. The boundary at $x=0$ is also the thermalizing wall with the temperature $T_{h}$ in both of the processes. If a particle with the mass $m$ collides with the thermalizing wall with the temperature $T$, its velocity is stochastically changed to a value $\boldsymbol{v}=\left(v_{x}, v_{y}\right)$ according to the distribution functions

$$
\begin{gathered}
P_{x}\left(v_{x}\right)=\frac{m}{k_{B} T}\left|v_{x}\right| \exp \left(-\frac{m v_{x}^{2}}{2 k_{B} T}\right), \\
\text { where }\left\{\begin{array}{l}
v_{x}>0 \text { at } x=0 \\
v_{x}<0 \text { at } x=L_{x},
\end{array}\right. \\
P_{y}\left(v_{y}\right)=\sqrt{\frac{m}{2 \pi k_{B} T}} \exp \left(-\frac{m v_{y}^{2}}{2 k_{B} T}\right),
\end{gathered}
$$

which ensure that the temperature of the equilibrium system becomes $T$. 
(a)
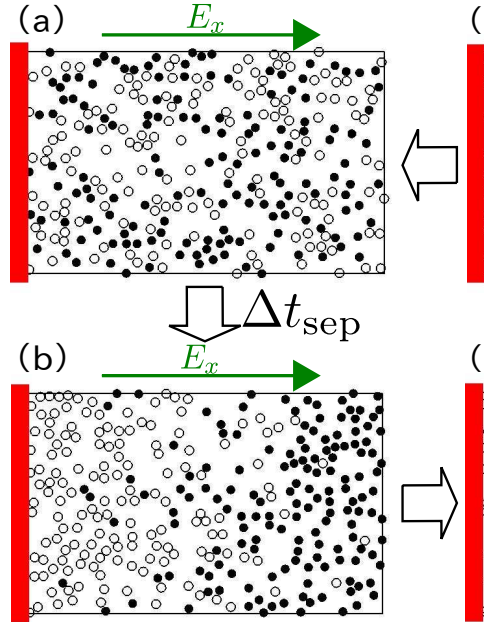

O:component A (d)

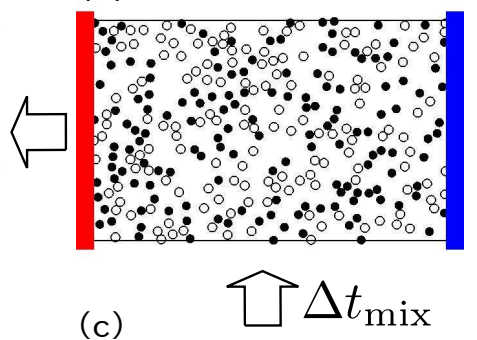

(c)

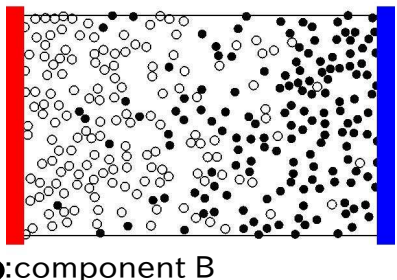

Fig. 2. Example of the snapshots of the system. (a) The beginning of the separating process. (b) The end of the separating process. (c) The beginning of the mixing process. (d) The end of the mixing process. The white disks and the black disks indicate the low-molecular-weight component $A$ and the highmolecular-weight component $B$, respectively.

In the following simulations, we use the scale units as $m_{A} \equiv 1, \sigma \equiv 1, \epsilon \equiv 1, q \equiv 1$, and $k_{B} \equiv 1$, which define the units of mass, length, energy, electric charge, and temperature, respectively. The time evolution of the system is performed by the velocity-Verlet scheme 19 with the time step $\delta t=0.0005$.

\subsection{Results of the simulation}

Figure 2 shows an example of the snapshots of the system. In the simulation, the system size is $L_{x} \times L_{y}=40 \times 25$, the numbers of the particles are $N_{A}=N_{B}=150$, the external field is $E=0.1$, and the temperatures of the heat baths are $T_{h}=1.01$ and $T_{c}=0.99$. Each particle of the components $A$ and $B$ has the mass $m_{A}=1$ and $m_{B}=10$, and the electric charge $q_{A}=-1$ and $q_{B}=1$, respectively.

From these snapshots, we can confirm that the components $A$ and $B$ are separated by applying the external field $E$ in the separating process and the components are diffusively mixed when the external field is turned off in the mixing process. This result can quantitatively be verified in Figure 3 which shows an example of the time evolution of the mole fraction profiles $x_{A}(x, t)$ in the mixing process and the separating process, where we calculated $x_{A}$ by dividing the system into 40 subsystems in the x-direciton.

Figure 4 depicts typical results of the time evolution of the temperature profiles $T(x, t)$ of the system, which is calculated from the kinetic energy as $T=\frac{1}{2 N_{x}} \sum_{j=1}^{N_{x}} m_{j} \boldsymbol{v}_{j}^{2}$, by using the same subsystems as in Figure 3 , where $N_{x}$ is the number of particles in the subsystem at position $x$, and $m_{j}$ and $\boldsymbol{v}_{j}$ are the mass and velocity of the $j$ th particle in that subsystem, respectively.
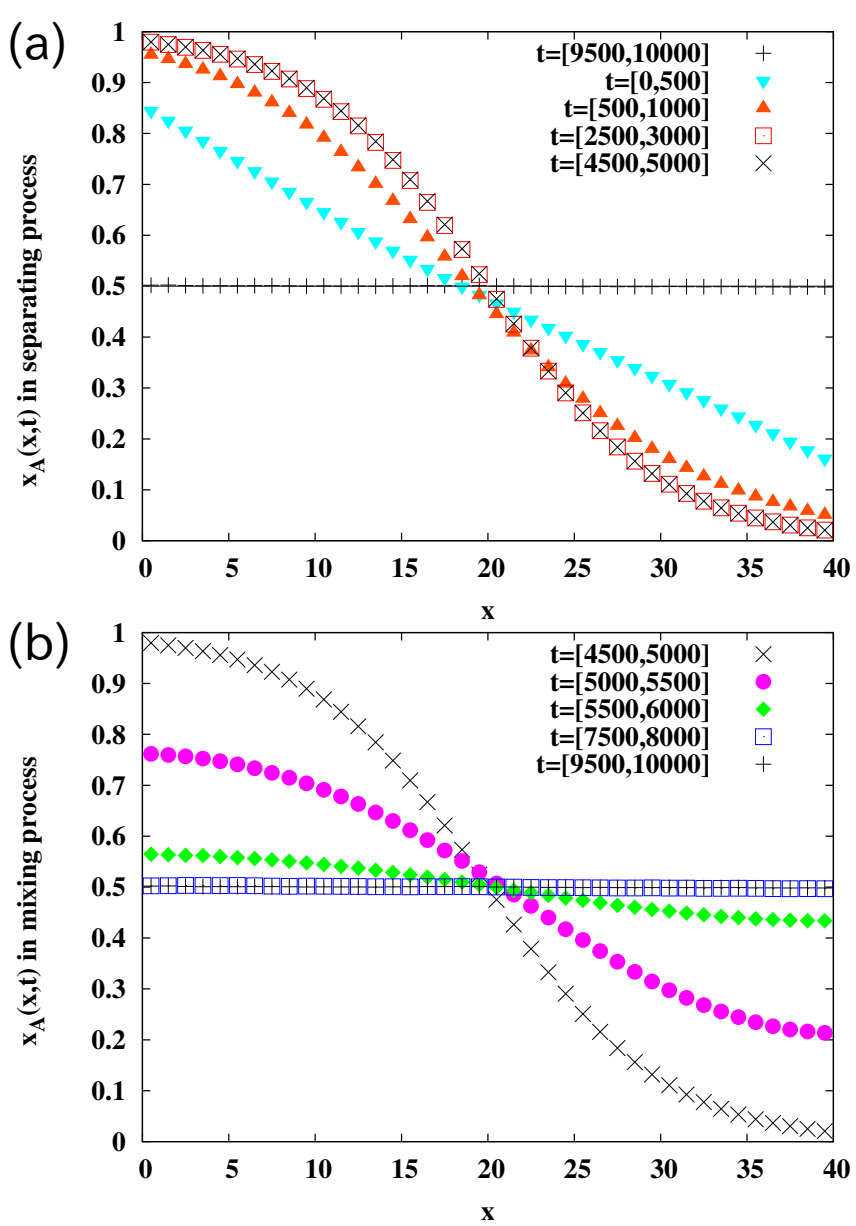

Fig. 3. The mole fraction profile $x_{A}(x, t)$ in the separating process $(0<t \leq 5000)$, and in the mixing process $(5000<t \leq$ $10000)$, with $\Delta t_{\mathrm{sep}}=\Delta t_{\mathrm{mix}}=5000$. A curve of $t=\left[t_{1}: t_{2}\right]$ means a profile averaged over the time between $t_{1} \leq t \leq t_{2}$. Furthermore, the MD data were averaged over 2640 cycles.

In the separating process, after the sudden increase of the temperature due to the heat produced from the work done by the external field $E_{x}$, the heat in the system gradually leaks into the heat bath with $T_{h}$, and then the total system reaches the equilibrium state at the temperature $T_{h}$. In contrast, in the mixing process, the system reaches a nonequilibrium steady state of heat conduction with a spatially linear temperature profile. We note that the temperature profile of the data $t=[5000,5500]$ in Figure $4 \mathrm{~b}$ can be explained as follows. At the early stage of the mixing process, in the middle region of the system, the Dufour effect due to the large mole-fraction gradient (see the data $\mathrm{t}=[4500,5000]$ in Fig. 3 $\mathrm{b}$ ) causes a large heat flow in the negative $x$-direction, while in the regions of both ends, the heat flow by the Dufour effect is small due to the small mole-fraction gradient. Accordingly, the temperature profile develops a maximum and a minimum near both ends, as shown by the data $t=[5000,5500]$ in Figure 4 $b$. Once the maximum and the minimum of the temperature profile are formed, the heat pump becomes functional by the heat flow in the negative $x$-direction due to the temper- 

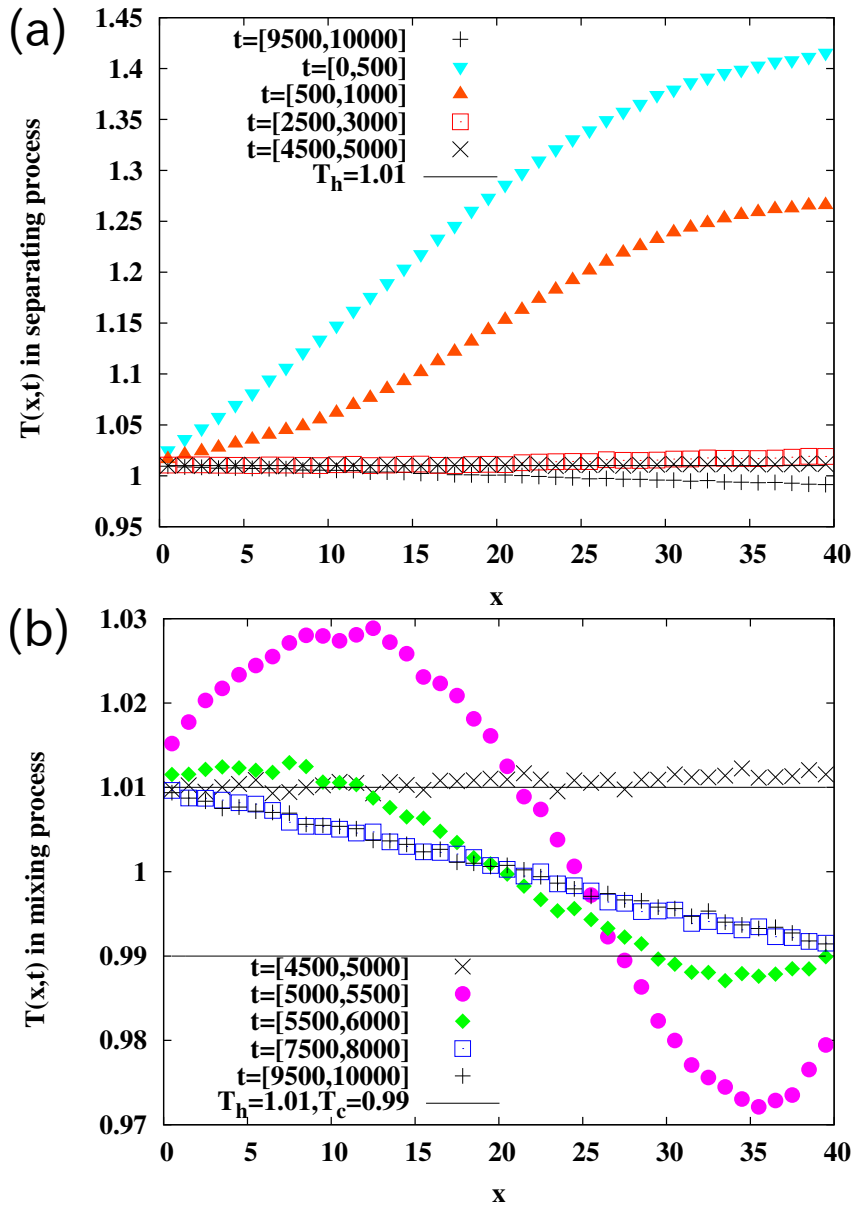

Fig. 4. The temperature profiles $T(x, t)$ in the separating process $(0<t \leq 5000)$, and in the mixing process $(5000<$ $t \leq 10000)$. A curve of $t=\left[t_{1}: t_{2}\right]$ is drawn following the same rule with Figure 3 . The solid lines denote the heat bath temperatures $T_{h}=1.01$ and $T_{c}=0.99$.

ature gradient near both ends at this early stage of the mixing process.

Figure 5 shows the time evolution of the number-density profiles of the particles $n(x, t)$. The peaks of $n(x, t)$ near the boundaries seem to be essentially the same phenomena as the particle adsorption at a hard wall reported in references [24,25]. We can find from Figure 5] that the profile $n(x, t)$ in the mixing process instantly reaches the steady profile compared with the mole fraction $x_{A}(x, t)$ in Figure 3 and the temperature $T(x, t)$ in Figure 4 . This result is assumed to hold in general for the theoretical analysis in Section 4.

In Figure 6] we measured the heat currents $\dot{Q}_{h}(t)$ flowing from the system into the heat bath with $T_{h}$ and $\dot{Q}_{c}(t)$ flowing from the heat bath with $T_{c}$ into the system. Here, we calculated $\dot{Q}_{\alpha}$ by accumulating over the unit time the kinetic energy change $\frac{m}{2}\left(v_{0}^{2}-v^{2}\right)$ at a particle collision with the thermalizing wall with $T_{\alpha}(\alpha=h, c)$, where $m$ is the mass of the particle and $v_{0}$ and $v$ are the velocities of the particle before and after the collision, respectively. We can see that $\dot{Q}_{h}(t)$ has a peak corresponding to the heat injection due to the external field in the separating
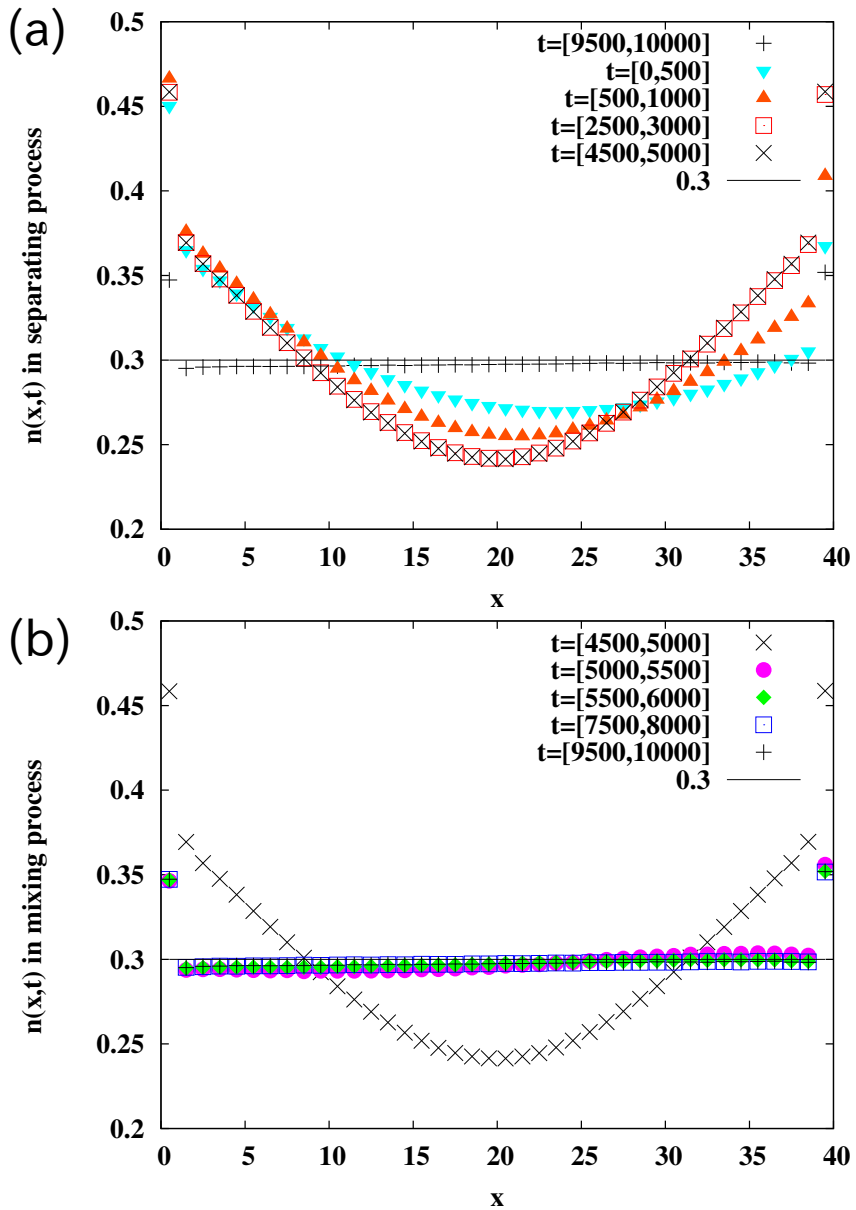

Fig. 5. The number-density profiles of the particles $n(x, t)$ in the separating process $(0<t \leq 5000)$, and in the mixing process $(5000<t \leq 10000)$. A curve of $t=\left[t_{1}: t_{2}\right]$ is drawn following the same rule with Figure 3 The solid line denotes the average number-density $\bar{n}=0.3$.

process, and the thermal equilibrium state of the total system is realized at last. The peaks of $\dot{Q}_{h}(t)$ and $\dot{Q}_{c}(t)$ in the mixing process which have a similar profile imply that the heat flows from the cold heat bath with $T_{c}$ toward the hot heat bath with $T_{h}$ through the system. Therefore we can see that a heat pump due to the Dufour effect is realized.

To confirm that our model is surely useful as a heat pump, we measured the cooling power $\overline{\dot{Q}}_{c}$ and the coefficient of performance $(\mathrm{COP}) \epsilon$ defined as

$$
\begin{gathered}
\overline{\dot{Q}}_{\alpha} \equiv \frac{1}{\tau_{1}-\tau_{0}} \int_{\tau_{0}}^{\tau_{1}} \dot{Q}_{\alpha}(t) d t \quad(\alpha=h, c), \\
\epsilon \equiv \frac{\overline{\dot{Q}}_{c}}{\overline{\bar{W}}},
\end{gathered}
$$

where $\tau_{0}$ is the relaxation time for the system to exhibit a steady cyclic state, and $\tau_{1}$ is chosen so that $\tau_{1}-\tau_{0}$ is an integer multiple of the cycle period $\Delta t_{\text {mix }}+\Delta t_{\text {sep }} \cdot \overline{\dot{W}}$ in equation (8) denotes the average power done by the external field $E_{x}(t)$ per unit time, which is calculated using the relation $\overline{\dot{W}}=\overline{\dot{Q}}_{h}-\overline{\dot{Q}}_{c}$. The cooling power $\overline{\dot{Q}}_{c}$ 


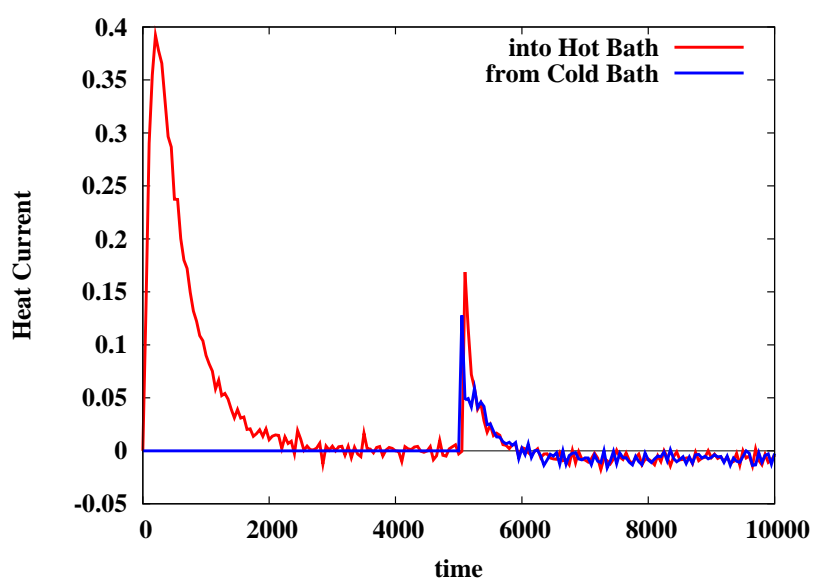

Fig. 6. Time dependence of the heat currents $\dot{Q}_{c}(t)$ flowing from the heat bath with $T_{c}$, and $\dot{Q}_{h}(t)$ flowing into the heat bath with $T_{h}$. The system is in separating process when $0<$ $t \leq 5000$, and in the mixing process when $5000<t \leq 10000$. The MD data were averaged over 2640 cycles.

and the COP $\epsilon$ should be positive for a useful heat pump. Figure 7 shows the $\delta T$-dependence of $\overline{\dot{Q}}_{c}$ and $\epsilon$, where $\delta T \equiv T_{h}-T_{c}$. While the cooling power and the COP are surely positive when $\delta T$ is small, they become negative when $\delta T$ is large, because the heat pumping by the Dufour effect cannot overcome the temperature gradient between the heat baths. Consequently, this numerical result implies that our model is useful as a heat pump when the temperature difference $\delta T$ is sufficiently small and $\Delta t_{\text {sep }}$ and $\Delta t_{\text {mix }}$ are suitably chosen.

\section{Theoretical analysis}

\subsection{Expressions for the Cooling Power and the COP}

First, we consider a simple case that the heat baths have the same temperature $T_{0}\left(=T_{h}=T_{c}\right)$, and assume that a process is switched to another process after the equilibrium state is realized, which means $\Delta t_{\text {sep }} \gg \tau_{\text {sep }}$ and $\Delta t_{\text {mix }} \gg \tau_{\text {mix }}$ where $\tau_{\text {sep }}$ and $\tau_{\text {mix }}$ are the relaxation times of the system to the steady states in the separating process and the mixing process, respectively. To obtain simple expressions for the cooling power and the COP, we assume that the mechanical equilibrium state (see Chap.V2 in Ref. [12) is instantly realized in the mixing process. This assumption means that the system satisfies $\nabla p=$ $n_{A} \boldsymbol{F}_{A}+n_{B} \boldsymbol{F}_{B}$ where $p$ is the pressure and $\boldsymbol{F}_{k}$ is the external force on each particle of component $k$. Therefore, the pressure gradient $\nabla p$ vanishes as

$$
\nabla p=0,
$$

in the mixing process where the electric field is turned off. Furthermore, we assume that the number-density profile of the particles $n(x, t)$ in the mixing process reaches the steady profile instantly compared with the mole fraction profile $x_{A}(x, t)$ and the temperature profile $T(x, t)$, which
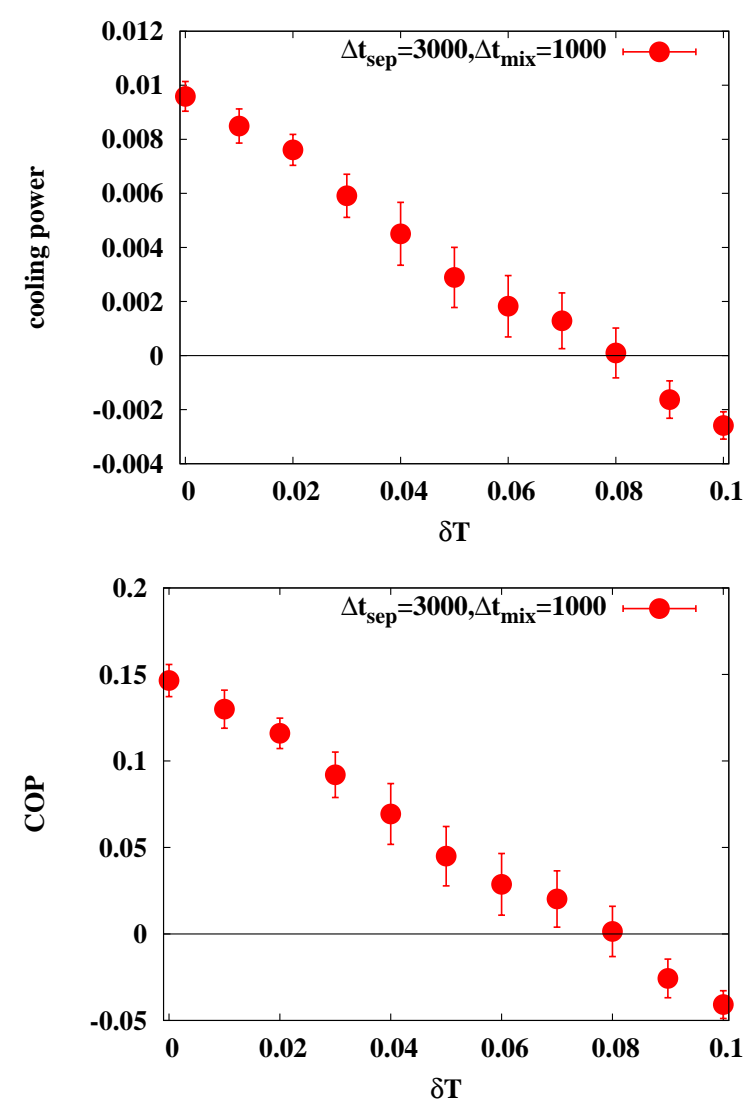

Fig. 7. The temperature difference $\delta T=T_{h}-T_{c}$ dependence of the cooling power and the COP, with the process durations $\Delta t_{\mathrm{sep}}=3000, \Delta t_{\mathrm{mix}}=1000$, and the temperatures $T_{h}=1+$ $\delta T / 2$ and $T_{c}=1-\delta T / 2$. The MD data were averaged over 345 cycles.

is confirmed to hold in our system from the numerical results in Figures 3.5. From this assumption $n(x, t)$ is approximately regarded as

$$
n(x, t)=\bar{n} \equiv N / V
$$

in the mixing process, where $N$ is the total number of particles in the system and $V$ is the volume of the system.

From the linear irreversible thermodynamics (see Chap. XI-7 in Ref. 12 ), when the system is uniform in the $y$ direction and the external field does not exist, the heat current $J_{Q}$ and the diffusion current $\bar{J}_{A}^{m}$ of the component $A$, which is defined as $\bar{J}_{k}^{m} \equiv n_{k}\left(v_{k}-v^{m}\right)$ where $v^{m} \equiv \sum_{k} x_{k} v_{k}$ is the mean velocity and $v_{k}$ is the velocity of the component $k$ in the $x$-direction, are written as

$$
\begin{aligned}
& J_{Q}=-\kappa \frac{\partial T}{\partial x}-n_{A} T D^{\prime \prime} \tilde{\mu}_{A A}^{x} \frac{\partial x_{A}}{\partial x} \\
& \bar{J}_{A}^{m}=-n x_{A} x_{B} D^{\prime} \frac{\partial T}{\partial x}-n D \frac{\partial x_{A}}{\partial x}
\end{aligned}
$$

where $D^{\prime}$ and $D$ denote the thermal diffusion coefficient and the diffusion coefficient, respectively, $\tilde{\mu}_{A}$ is the chemical potential per particle of the component $A$, and $\tilde{\mu}_{A A}^{x} \equiv$ $\left(\partial \tilde{\mu}_{A} / \partial x_{A}\right)_{T, p}$. Equations (11) and (12) can be derived 
by taking the thermodynamic forces as $-\nabla T / T^{2}$ and $-\tilde{\mu}_{A A}^{x}\left(\nabla x_{A}\right) /\left(x_{B} T\right)$. Then, the coefficients of the Onsager relations

$$
\begin{aligned}
J_{Q} & =-L_{q q} \frac{1}{T^{2}} \frac{\partial T}{\partial x}-L_{q A} \frac{\tilde{\mu}_{A A}^{x}}{x_{B} T} \frac{\partial x_{A}}{\partial x} \\
\bar{J}_{A}^{m} & =-L_{A q} \frac{1}{T^{2}} \frac{\partial T}{\partial x}-L_{A A} \frac{\tilde{\mu}_{A A}^{x}}{x_{B} T} \frac{\partial x_{A}}{\partial x}
\end{aligned}
$$

are written as $L_{q q}=\kappa T^{2}, L_{q A}=n x_{A} x_{B} T^{2} D^{\prime \prime}, L_{A q}=$ $n x_{A} x_{B} T^{2} D^{\prime}$ and $L_{A A}=n x_{B} T D / \tilde{\mu}_{A A}^{x}$, thus the Onsager reciprocal relation leads to $D^{\prime \prime}=D^{\prime}$. Additionally, if $v^{m}=$ 0 holds, the time evolution equations of $T$ and $x_{A}$ written as

$$
\begin{aligned}
c_{p} \frac{\partial T}{\partial t} & =\frac{\partial}{\partial x}\left\{\kappa \frac{\partial T}{\partial x}+n_{A} T D^{\prime \prime} \tilde{\mu}_{A A}^{x} \frac{\partial x_{A}}{\partial x}\right\}, \\
n \frac{\partial x_{A}}{\partial t} & =\frac{\partial}{\partial x}\left\{n x_{A} x_{B} D^{\prime} \frac{\partial T}{\partial x}+n D \frac{\partial x_{A}}{\partial x}\right\},
\end{aligned}
$$

which can be derived from the conservation laws of energy and mass (see Chap.XI-7 in Ref. 12]), respectively. Here, $c_{p}$ is the specific heat at constant pressure per unit volume.

The time evolution equations of $x_{A}$ and $T$ in the mixing process can be derived from equations (15) and (16) by using $\partial p / \partial x=\partial n / \partial x=0$ (in Eqs. (9) and (10)), and then can be simplified by neglecting the second-order terms of $\partial T / \partial x$ and $\partial x_{A} / \partial x$, as

$$
\begin{aligned}
c_{p} \frac{\partial T}{\partial t} & =l_{11}(x, t) \frac{\partial^{2} T}{\partial x^{2}}+l_{12}(x, t) \frac{\partial^{2} x_{A}}{\partial x^{2}}, \\
n \frac{\partial x_{A}}{\partial t} & =l_{21}(x, t) \frac{\partial^{2} T}{\partial x^{2}}+l_{22}(x, t) \frac{\partial^{2} x_{A}}{\partial x^{2}},
\end{aligned}
$$

where $l_{11} \equiv \kappa, l_{12} \equiv n_{A} \tilde{\mu}_{A A}^{x} T D^{\prime \prime}, l_{21} \equiv n x_{A} x_{B} D^{\prime}$, and $l_{22} \equiv n D$. We note that the coefficients $l_{i j}$ depend on the position $x$ and the time $t$ through $p, T, x_{A}$ and $n$. These time evolution equations should be solved under the boundary conditions

$$
\begin{gathered}
\bar{J}_{A}^{m}(0, t)=\bar{J}_{A}^{m}\left(L_{x}, t\right)=0, \\
T(0, t)=T\left(L_{x}, t\right)=T_{0},
\end{gathered}
$$

using equation (12). Since $\Delta t_{\text {sep }} \gg \tau_{\text {sep }}$, the initial condition of the mixing process is written as

$$
T(x, 0)=T_{0}, \quad x_{A}(x, 0)=x_{A}^{E}(x),
$$

where $x_{A}^{E}(x)$ denotes the mole fraction profile of the equilibrium state in the end of the separating process with the external field $E$ and we note that $t=0$ is chosen as the beginning of the mixing process unlike Figures 3-6. Similarly, because of $\Delta t_{\text {mix }} \gg \tau_{\text {mix }}$, the profiles of the mole fraction $x_{A}$ and the temperature $T$ in the end of the mixing process are written as

$$
T\left(x, \Delta t_{\text {mix }}\right)=T_{0}, \quad x_{A}\left(x, \Delta t_{\text {mix }}\right)=\bar{x}_{A},
$$

where $\bar{x}_{A} \equiv N_{A} / N$ is the mean mole fraction in the system.

The cooling power (7) is expressed as

$$
\bar{Q}_{c} \equiv \frac{-1}{\Delta t_{\mathrm{sep}}+\Delta t_{\mathrm{mix}}} \int_{0}^{\Delta t_{\mathrm{mix}}} L_{y} J_{Q}\left(L_{x}, t\right) d t,
$$

after the steady cyclic state is established. By using equations (11) and (12) with the coefficients $l_{i j}$ and the boundary condition (19), we can obtain

$$
\overline{\dot{Q}}_{c}=\frac{L_{y} \int_{0}^{\Delta t_{\mathrm{mix}}}\left(l_{11}-l_{12} \frac{l_{21}}{l_{22}}\right) \frac{\partial T}{\partial x}\left(L_{x}, t\right) d t}{\Delta t_{\mathrm{sep}}+\Delta t_{\mathrm{mix}}} .
$$

To obtain the expression for the COP, we write equation (8) as

$$
\epsilon=\frac{\overline{\dot{Q}}_{c}}{W_{E} /\left(\Delta t_{\mathrm{sep}}+\Delta t_{\mathrm{mix}}\right)}
$$

using the relation $\overline{\dot{W}}=W_{E} /\left(\Delta t_{\text {sep }}+\Delta t_{\text {mix }}\right)$, where $W_{E}$ denotes the total work done by the external field $E_{x}=E$ in the separating process. The work $W_{E}$ is written as

$$
W_{E}=\psi_{E}^{\text {initial }}-\psi_{E}^{\text {final }},
$$

where $\psi_{E}^{\text {initial }}$ and $\psi_{E}^{\text {final }}$ are the potential energies due to the electric field $E_{x}=E$ in the initial and final states, respectively, of the separating process (see Appendix (B). Using the profiles $x_{A}(x)$ and $n(x)$ of the system, the potential $\psi_{E}$ is given by

$$
\psi_{E}\left[x_{A}(x), n(x)\right]=q E L_{y} \int_{0}^{L_{x}} n(x)\left(2 x_{A}(x)-1\right) x d x .
$$

Since, in the separating process, the initial profiles of $x_{A}(x)$ and $n(x)$ are $\bar{x}_{A}$ and $\bar{n}$, respectively, and the final profiles are $x_{A}^{E}(x)$ and $n^{E}(x)$, where $n^{E}(x)$ is defined similarly to $x_{A}^{E}(x)$ below equation (21), equation (26) becomes

$$
\begin{aligned}
W_{E}=-q E & L_{y} \int_{0}^{L_{x}}\left\{\delta n^{E}(x)\left(2 \bar{x}_{A}-1\right)\right. \\
& \left.+2 \bar{n} \delta x_{A}^{E}(x)+2 \delta n^{E}(x) \delta x_{A}^{E}(x)\right\} x d x
\end{aligned}
$$

where we defined $\delta x_{A}^{E}(x) \equiv x_{A}^{E}(x)-\bar{x}_{A}$ and $\delta n^{E}(x) \equiv$ $n^{E}(x)-\bar{n}$. Therefore, by substituting equation (28) into equation (25), the expression for the COP is written as

$$
\epsilon=\frac{\int_{0}^{\Delta t_{\text {mix }}}\left(-l_{11}+l_{12} \frac{l_{21}}{l_{22}}\right) \frac{\partial T}{\partial x}\left(L_{x}, t\right) d t}{q E \int_{0}^{L_{x}}\left\{\left(2 \bar{x}_{A}-1\right) \delta n^{E}(x)+2 \bar{n} \delta x_{A}^{E}(x)+2 \delta n^{E}(x) \delta x_{A}^{E}(x)\right\} x d x}
$$




\subsection{Approximate calculation of the Cooling Power and the COP}

We make two assumptions to calculate $\overline{\dot{Q}}_{c}$ and $\epsilon$ approximately. The first assumption is that $\partial T / \partial x, \partial x_{A} / \partial x$ and $E$ are very small so that the coefficients $l_{i j}, c_{p}$ and $n$ in the time evolution equations (17) and (18) approximately depend only on the average values over the system, not on the time and the position. Under this assumption, we write $l_{i j}, c_{p}$ and $n$ as $\bar{l}_{i j}, \bar{c}_{p}$ and $\bar{n}$, respectively, in the following. Therefore, we can linearize equations (17) and (18) with the constants $\bar{l}_{i j}, \bar{c}_{p}$ and $\bar{n}$ as

$$
\begin{aligned}
& \bar{c}_{p} \frac{\partial T}{\partial t}(x, t)=\bar{l}_{11} \frac{\partial^{2} T}{\partial x^{2}}(x, t)+\bar{l}_{12} \frac{\partial^{2} x_{A}}{\partial x^{2}}(x, t), \\
& \bar{n} \frac{\partial x_{A}}{\partial t}(x, t)=\bar{l}_{21} \frac{\partial^{2} T}{\partial x^{2}}(x, t)+\bar{l}_{22} \frac{\partial^{2} x_{A}}{\partial x^{2}}(x, t) .
\end{aligned}
$$

We can calculate the cooling power (24) by solving these time evolution equations (30) and (31) of the mixing process without using the similar equations of the separating process, because the heat does not flow from the cold heat bath in the separating process. The second assumption is that the mixture can be regarded as an ideal gas when the system is in the equilibrium state. Using the second assumption and the equilibrium statistical mechanics, $\delta n^{E}(x)$ and $\delta x_{A}^{E}(x)$ defined below equation (28) can be calculated as

$$
\begin{aligned}
\delta n^{E}(x) & =\frac{\beta E q}{L_{y}} \frac{N_{A} e^{\beta E q\left(\frac{L_{x}}{2}-x\right)}+N_{B} e^{-\beta E q\left(\frac{L_{x}}{2}-x\right)}}{e^{\beta E q \frac{L_{x}}{2}}-e^{-\beta E q \frac{L_{x}}{2}}}-\bar{n} \\
& \simeq\left(2 \bar{n}_{A}-\bar{n}\right) \beta E q\left(\frac{L_{x}}{2}-x\right), \\
\delta x_{A}^{E}(x) & =\frac{N_{A} e^{\beta E q\left(\frac{L_{x}}{2}-x\right)}}{N_{A} e^{\beta E q\left(\frac{L_{x}}{2}-x\right)}+N_{B} e^{-\beta E q\left(\frac{L_{x}}{2}-x\right)}}-\bar{x}_{A} \\
& \simeq 2 \bar{x}_{A}\left(1-\bar{x}_{A}\right) \beta E q\left(\frac{L_{x}}{2}-x\right),
\end{aligned}
$$

where $\beta \equiv 1 / k_{B} \bar{T}$ and $\bar{T}=T_{0}$, and we expanded the equations up to the first order of $E$. From the assumption of ideal gas, we can obtain $\tilde{\mu}_{A A}^{x}=k_{B} T / x_{A}$, therefore

$$
\bar{l}_{12}=k_{B} \bar{T}^{2} \bar{n} D^{\prime \prime} \text {. }
$$

Similarly, we can obtain the relations

$$
\begin{aligned}
& \bar{l}_{21}=\bar{n} \bar{x}_{A} \bar{x}_{B} D^{\prime}, \\
& \bar{l}_{22}=\bar{n} D .
\end{aligned}
$$

We next give the integral $\int_{0}^{\Delta t_{\text {mix }}}(\partial T / \partial x)\left(L_{x}, t\right) d t$ in the expression for the cooling power (24) By eliminating $\partial^{2} x_{A} / \partial x^{2}$ from equations (30) and (31), we obtain

$$
\bar{c}_{p} \frac{\partial T}{\partial t}=\bar{l}_{1}^{\prime} \frac{\partial^{2} T}{\partial x^{2}}+\bar{l}_{2}^{\prime} \frac{\partial x_{A}}{\partial t},
$$

where

$$
\begin{aligned}
& \bar{l}_{1}^{\prime} \equiv \bar{l}_{11}-\bar{l}_{12} \bar{l}_{21} / \bar{l}_{22}, \\
& \bar{l}_{2}^{\prime} \equiv \bar{l}_{12} \bar{n} / \bar{l}_{22},
\end{aligned}
$$

are introduced for simplicity. By integrating equation (39) with respect to the time $t$ on $\left[0, \Delta t_{\text {mix }}\right]$, we obtain

$$
0=\bar{l}_{1}^{\prime} \frac{\partial^{2}}{\partial x^{2}} \int_{0}^{\Delta t_{\mathrm{mix}}} T(x, t) d t+\bar{l}_{2}^{\prime}\left(-\delta x_{A}^{E}(x)\right) .
$$

The above equation can be integrated with respect to $x$ by substituting $\delta x_{A}^{E}(x)$ of equation (35) into equation (42) and using the boundary condition (20). Then, we obtain

$$
\begin{aligned}
\int_{0}^{\Delta t_{\mathrm{mix}}} T(x, t) d t & =\frac{2 \bar{l}_{2}^{\prime} \bar{x}_{A}\left(1-\bar{x}_{A}\right) \beta E q}{\bar{l}_{1}^{\prime}} \\
& \times\left(-\frac{x^{3}}{6}+\frac{L_{x}}{4} x^{2}-\frac{L_{x}^{2}}{12} x\right)+T_{0} \Delta t_{\mathrm{mix}} .
\end{aligned}
$$

Therefore, the cooling power is written as

$$
\overline{\dot{Q}}_{c}=\frac{-k_{B} \bar{T}^{2} \bar{n} D^{\prime \prime} \bar{x}_{A}\left(1-\bar{x}_{A}\right) \beta E q L_{y} L_{x}^{2}}{6 D\left(\Delta t_{\mathrm{sep}}+\Delta t_{\mathrm{mix}}\right)} .
$$

By substituting equations (33) and (35) into equation (28), and expanding up to the second order of $E$, the work $W_{E}$ becomes

$$
W_{E}=\frac{L_{y} L_{x}^{3} \beta(q E)^{2} \bar{n}}{12}
$$

Consequently, the COP in equation (25) is written as

$$
\epsilon=\frac{-2 k_{B} \bar{T}^{2} D^{\prime \prime} \bar{x}_{A}\left(1-\bar{x}_{A}\right)}{L_{x} q E D} .
$$

\subsection{Numerical confirmation}

To compare the theoretical results (44) and (46) with the MD results, the transport coefficients such as $D$ and $D^{\prime \prime}$ need to be determined. It is convenient to introduce the thermal diffusion ratio $k_{T}$ defined as

$$
k_{T} \equiv \bar{T} \bar{x}_{A} \bar{x}_{B} \frac{D^{\prime}}{D},
$$

because our main results (44) and (46) can be rewritten with only $k_{T}$ instead of $D$ and $D^{\prime \prime}\left(=D^{\prime}\right)$ as

$$
\begin{aligned}
\overline{\dot{Q}}_{c} & =\frac{-k_{T} N E q L_{x}}{6\left(\Delta t_{\mathrm{sep}}+\Delta t_{\mathrm{mix}}\right)}, \\
\epsilon & =\frac{-2 k_{B} k_{T} \bar{T}}{L_{x} q E},
\end{aligned}
$$

respectively. 


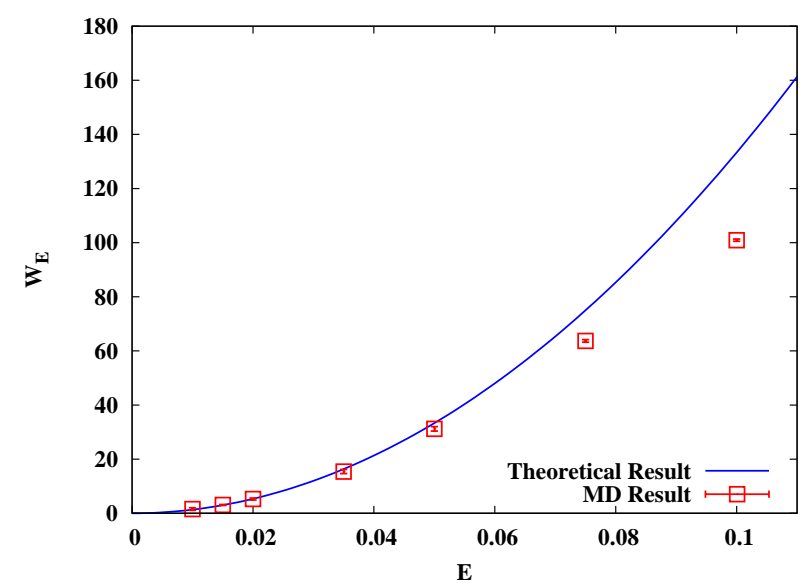

Fig. 8. Comparison between the theoretical result 445) and the MD result of the work $W_{E}$ done by the external field $E$ to the system. The MD data were averaged over 2000-7500 cycles.

$k_{T}$ can approximately be calculated from the ChapmanEnskog theory (see Appendix (A). We numerically calculated the two-dimensional expression for $k_{T}$ in the first order approximation as

$$
k_{T} \simeq-0.13657
$$

using the parameters $m_{A}=1, m_{B}=10, \bar{T}=1, \bar{x}_{A}=$ $\bar{x}_{B}=0.5$, and $Y=10^{5}$ of the Herzian potential.

In the MD simulations in this section, the numbers of particles are changed to $N_{A}=N_{B}=50$ so that the number-density of the particles in the system becomes adequately dilute, which is assumed in the Chapman-Enskog theory. The calculation of equation (50) is also valid for these new parameters. $\Delta t_{\text {sep }}$ and $\Delta t_{\text {mix }}$ are fixed to 10000 and 5000, respectively, so that the assumption of $\Delta t_{\text {sep }} \gg$ $\tau_{\text {sep }}$ and $\Delta t_{\text {mix }} \gg \tau_{\text {mix }}$ is satisfied. All other parameters such as the system size are identical with Section 2 .

Figure 8 shows the numerical result of the work $W_{E}$ done by the external field $E_{x}=E$ as varying $E$, together with the theoretical result (45). From Figure 8, we can see that the MD data deviate from the theoretical curve when $0.07 \lesssim E$. This result implies that the assumption of small $E$ in our theory is not satisfied when $0.07 \lesssim E$ and the consequent results of the theory may not be accurate. This is because the number-density in some parts of the mixture becomes high and the mixture deviates from ideal gas when the external field $E$ is large.

The theoretical results of the cooling power $\overline{\dot{Q}}_{c}$ and the COP $\epsilon$ in equations (48) and (49) using the value of equation (50) are compared with the MD data in Figure 9] We can confirm a good agreement between the theory and the MD data in the region $E \lesssim 0.05$, but a small discrepancy in the region $0.07 \lesssim E$ where the assumption of small $E$ may not be satisfied. Therefore, we conclude that the validity of our theoretical analysis of the heat pump model is verified in the case of $T_{h}=T_{c}$.

\subsection{The case of $T_{h} \neq T_{c}$}

Finally, we show that the theoretical analysis in Sections 4.1 and 4.2 can be generalized to the case of $T_{h} \neq T_{c}$. We consider the case that the temperature difference of the heat baths $\delta T \equiv T_{h}-T_{c}$ is very small, and $\Delta t_{\text {sep }} \gg$ $\tau_{\text {sep }}$ and $\Delta t_{\text {mix }} \gg \tau_{\text {mix }}$ are satisfied. The time evolution equations (17) and (18) in the mixing process hold even in this case, and we assume that the linear approximation in equations (30) and (31) is also valid. The boundary condition (19) is unchanged, but equation (20) should be changed to

$$
T(0, t)=T_{\mathrm{h}}, \quad T\left(L_{x}, t\right)=T_{\mathrm{c}} .
$$

The initial conditions of $T(x, t)$ and $x_{A}(x, t)$ in the mixing process are

$$
T(x, 0)=T_{\mathrm{h}}, \quad x_{A}(x, 0)=x_{A}^{E}(x),
$$

respectively.

The profiles $T(x, t)$ and $x_{A}(x, t)$ in the end of the mixing process in equation (22) become

$$
T\left(x, \Delta t_{\text {mix }}\right)=T^{\delta T}(x), \quad x_{A}\left(x, \Delta t_{\text {mix }}\right)=x_{A}^{\delta T}(x),
$$
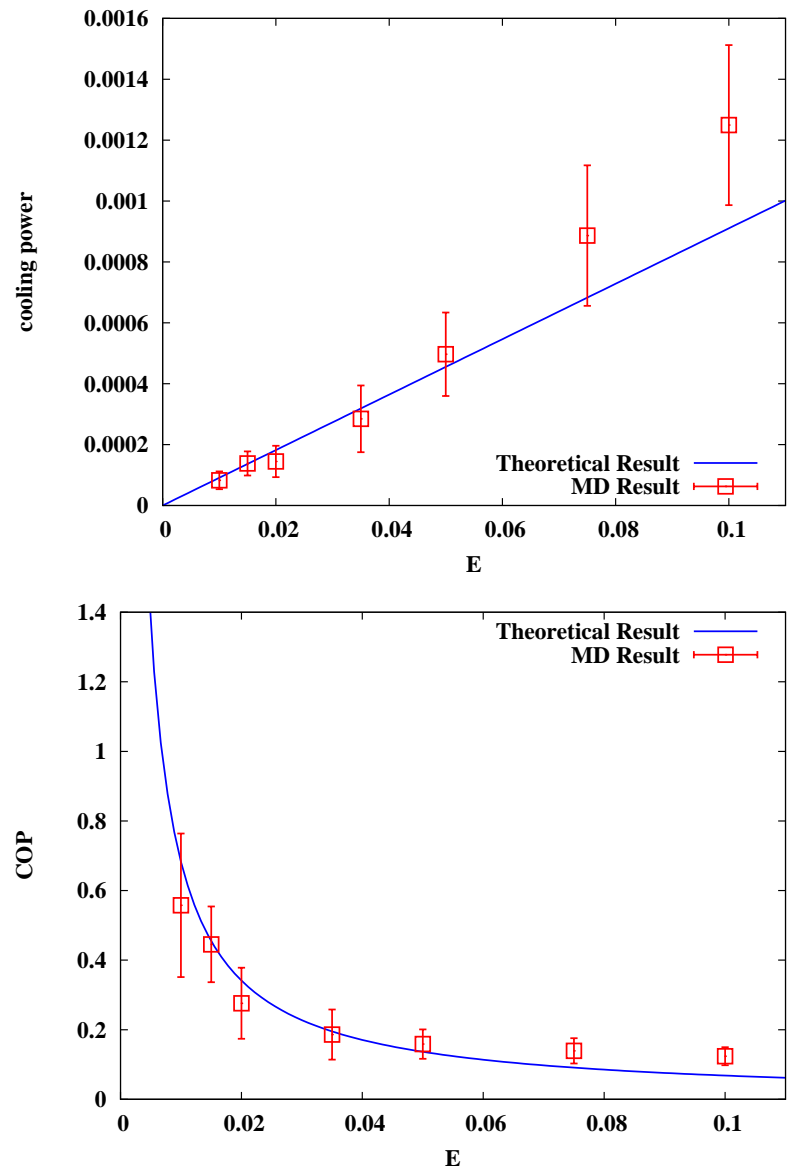

Fig. 9. Comparison between the theoretical results (48) and (49) and the MD results of the cooling power $\bar{Q}_{c}$ and the COP $\epsilon$, respectively. The MD data were averaged over 2000-7500 cycles. 
where $T^{\delta T}(x)$ and $x_{A}^{\delta T}(x)$ denote the temperature and the mole fraction profiles, respectively, of the steady heatconduction state in the mixing process when the temperature difference between the heat baths $\delta T$ exists. In the steady state of the mixing process, the temperature profile $T^{\delta T}(x)$ is written as

$$
T^{\delta T}(x)=-\frac{\delta T}{L_{x}} x+T_{\mathrm{h}},
$$

which can be derived from the time evolution equations (30) and (31) and the boundary condition (51). To determine the mole fraction profile $x_{A}^{\delta T}(x)$, we need an additional assumption, besides the assumptions in Sections 4.1 and 4.2 that each local subsystems of the mixture can be regarded as equilibrium ideal gas in that subsystem when the system is in the steady state of the mixing process. Using the equation of state of ideal gas, we can write

$$
n^{\delta T}(x)=\frac{p(x)}{k_{B} T^{\delta T}(x)} \simeq \frac{p(x)}{k_{B} T_{\mathrm{h}}}\left(1+\frac{\delta T}{T_{\mathrm{h}} L_{x}} x\right),
$$

where $p(x)$ is the pressure profile, $n^{\delta T}(x)$ denotes the number-density profile of the steady state in the mixing process, and we neglected the terms $\mathcal{O}\left(\delta T^{2}\right)$. Using the assumption of the mechanical equilibrium state $\nabla p=0$ in Section 4.1 and the relation $N=\int_{0}^{L_{x}} L_{y} n^{\delta T}(x) d x$, equation (55) can be rewritten as

$$
n^{\delta T}(x)=\bar{n}+\frac{\bar{n} \delta T}{\bar{T} L_{x}}\left(x-\frac{L_{x}}{2}\right),
$$

where $\bar{T} \equiv\left(T_{h}+T_{c}\right) / 2$. When the system is in the steady state, the linear relation (12) becomes

$$
0=-\bar{l}_{21} \frac{\partial T^{\delta T}}{\partial x}(x)-\bar{l}_{22} \frac{\partial x_{A}^{\delta T}}{\partial x}(x) .
$$

Thus, from equation (54) and the relation

$$
N_{A}=\int_{0}^{L_{x}} L_{y} n^{\delta T}(x) x_{A}^{\delta T}(x) d x
$$

the mole fraction profile $x_{A}^{\delta T}(x)$ is written as

$$
x_{A}^{\delta T}(x)=\frac{\bar{x}_{A} \bar{x}_{B} D^{\prime} \delta T}{D L_{x}}\left(x-\frac{L_{x}}{2}\right)+\bar{x}_{A},
$$

where we use equations (37) and (38)

The cooling power $\overline{\dot{Q}}_{c}$ can be calculated in the same way as in Sections 4.1 and 4.2 , but the condition $\delta T \neq 0$ changes equation (42) to

$$
\begin{aligned}
\bar{c}_{p}\left(T^{\delta T}(x)-T_{\mathrm{h}}\right)= & \bar{l}_{1}^{\prime} \frac{\partial^{2}}{\partial x^{2}} \int_{0}^{\Delta t_{\mathrm{mix}}} T(x, t) d t \\
& \quad \bar{l}_{2}^{\prime}\left(\delta x_{A}^{\delta T}(x)-\delta x_{A}^{E}(x)\right),
\end{aligned}
$$

where $\delta x_{A}^{\delta T}(x) \equiv x_{A}^{\delta T}(x)-\bar{x}_{A}$. Since the expression (24) is valid even in the present case, the cooling power is obtained

as

$$
\overline{\dot{Q}}_{c}=\frac{-L_{y}}{6\left(\Delta t_{\mathrm{sep}}+\Delta t_{\mathrm{mix}}\right)}\left\{2 \bar{c}_{p} \delta T L_{x}+\frac{\bar{l}_{2}^{\prime} \bar{x}_{A} \bar{x}_{B} D^{\prime \prime} \delta T L_{x}}{2 D}+\bar{l}_{2}^{\prime} \bar{x}_{A} \bar{x}_{B} \beta\left(1-\frac{\delta T}{2 \bar{T}}\right) E q L_{x}^{2}+\frac{6 \bar{l}_{1}^{\prime} \delta T \Delta t_{\text {mix }}}{L_{x}}\right\},
$$

by solving the differential equation (60) for $\int_{0}^{\Delta t_{\text {mix }}} T(x, t) d t$. We note that $\bar{l}_{1}^{\prime}$ in equation (40) is positive since $L_{q A}^{2}<L_{q q} L_{A A}[26$.

In the case of $\delta T \neq 0$, the expression for $W_{E}$ in equation (28) becomes

$$
\begin{aligned}
W_{E}=\psi_{E}\left[\bar{x}_{A}+\delta x_{A}^{\delta T}(x), \bar{n}+\delta n^{\delta T}(x)\right] \\
\quad-\psi_{E}\left[\bar{x}_{A}+\delta x_{A}^{E}(x), \bar{n}+\delta n^{E}(x)\right] \\
=q E L_{y} \int_{0}^{L_{x}}\left\{\left(\delta n^{\delta T}(x)-\delta n^{E}(x)\right)\left(2 \bar{x}_{A}-1\right)\right. \\
\left.+2 \bar{n}\left(\delta x_{A}^{\delta T}(x)-\delta x_{A}^{E}(x)\right)+\mathcal{O}\left(\delta T^{2}\right)+\mathcal{O}\left(E^{2}\right)\right\} x d x
\end{aligned}
$$

where $\delta n^{\delta T}(x) \equiv n^{\delta T}(x)-\bar{n}$. By substituting equations (33), (35), (56) and (59) into equation (63), we can obtain

$$
\begin{array}{r}
W_{E} \simeq \frac{\bar{n} q E L_{y} L_{x}^{2}}{12}\left\{\frac{\left(2 \bar{x}_{A}-1+2 \bar{x}_{A} \bar{x}_{B} \bar{T} D^{\prime} / D\right) \delta T}{\bar{T}}\right. \\
\left.+\beta\left(1-\frac{\delta T}{2 \bar{T}}\right) E q L_{x}\right\}
\end{array}
$$

Substituting equations (61) and (64) into equation (25), we finally obtain the COP as

$$
\epsilon=\frac{-2}{\bar{n} q E L_{x}^{2}} \frac{2 \bar{c}_{p} \delta T L_{x}+\frac{\bar{l}_{2}^{\prime} \bar{x}_{A} \bar{x}_{B} D^{\prime \prime} \delta T L_{x}}{2 D}+\bar{l}_{2}^{\prime} \bar{x}_{A} \bar{x}_{B} \beta\left(1-\frac{\delta T}{2 \bar{T}}\right) E q L_{x}^{2}+\frac{6 \bar{l}_{1}^{\prime} \delta T \Delta t_{\mathrm{mix}}}{L_{x}}}{\frac{\left(2 \bar{x}_{A}-1+2 \bar{x}_{A} \bar{x}_{B} \bar{T} D^{\prime} / D\right) \delta T}{\bar{T}}+\beta\left(1-\frac{\delta T}{2 \bar{T}}\right) E q L_{x}} .
$$


Since $\bar{l}_{1}^{\prime}>0$, equation (65) means that the longer $\Delta t_{\text {mix }}$ is, the lower $\epsilon$ becomes because the heat begins to flow in the reverse direction after a temperature gradient is established due to the temperature difference of the heat baths.
Finally, we compare the theoretical results in this section with the MD results. By using equations (40) and (41), equations (61) and (65) can be rewritten as

$$
\begin{aligned}
\overline{\dot{Q}}_{c} & =\frac{-L_{y}}{6\left(\Delta t_{\mathrm{sep}}+\Delta t_{\mathrm{mix}}\right)}\left[\left\{2 \bar{c}_{p} L_{x}+\frac{k_{B} \bar{n} k_{T}^{2} L_{x}}{2 \bar{x}_{A} \bar{x}_{B}}-\frac{\bar{n} k_{T} E q L_{x}^{2}}{2 \bar{T}}+\frac{6 \lambda \Delta t_{\mathrm{mix}}}{L_{x}}\right\} \delta T+\bar{n} k_{T} E q L_{x}^{2}\right], \\
\epsilon & =\frac{-2}{\bar{n} q E L_{x}^{2}} \frac{\left\{2 \bar{c}_{p} L_{x}+\frac{k_{B} \bar{n} k_{T}^{2} L_{x}}{2 \bar{x}_{A} \bar{x}_{B}}-\frac{\bar{n} k_{T} E q L_{x}^{2}}{2 \bar{T}}+\frac{6 \lambda \Delta t_{\text {mix }}}{L_{x}}\right\} \delta T+\bar{n} k_{T} E q L_{x}^{2}}{\left\{2 \bar{x}_{A}-1+2 k_{T}-\frac{\beta E q L_{x}}{2}\right\} \frac{\delta T}{\bar{T}}+\beta E q L_{x}},
\end{aligned}
$$

respectively, where we introduced the coefficient $\lambda$ defined as

$$
\lambda=\kappa-\bar{n} k_{B} \bar{T}^{2} \bar{x}_{A} \bar{x}_{B} \frac{D^{\prime 2}}{D},
$$

which can be calculated in the first order approximation as

$$
\lambda \simeq 0.419877
$$

by using its microscopic expression A.13 with the same parameters as used in equation (50). Figure 10 shows the MD results of the cooling power and the COP as varying the temperature difference $\delta T$, together with the theoretical results (66) and (67) using equation (69) and $\bar{c}_{p}=2 k_{B} \bar{n}$, which is the two-dimensional ideal-gas value. In the MD simulation in Figure 10, the external field $E$ was changed to $E=0.035$ from $E=0.1$ of Figure 7 because our theory is valid when $E$ is sufficiently small. From this figure, we can see that the theory agrees with the MD data in the region of small $\delta T$, which shows that our theory is valid not only in the case of $\delta T=0$ in Figure 9 but also in the case of $\delta T \neq 0$.

\section{Summary}

We proposed a heat pump model utilizing the Dufour effect and studied it by using the MD simulation and the linear irreversible thermodynamics. This model consists of the separating process in which the mixture is separated by the external electric field, and the mixing process in which the Dufour effect occurs. Using the MD simulation, we calculated the cooling power and the COP of the model as in Figure 7 and numerically confirmed its usefulness as a heat pump. Next, we theoretically calculated the cooling power and the COP as equations (48) and (49), especially in the simplest case of $T_{h}=T_{c}, \Delta t_{\text {sep }} \gg \tau_{\text {sep }}$ and $\Delta t_{\text {mix }} \gg$ $\tau_{\text {mix }}$, and we confirmed a good agreement between the theoretical and MD results. Furthermore, we showed that our theory is generalized to the case of $T_{h} \neq T_{c}$ and is valid also in that case.
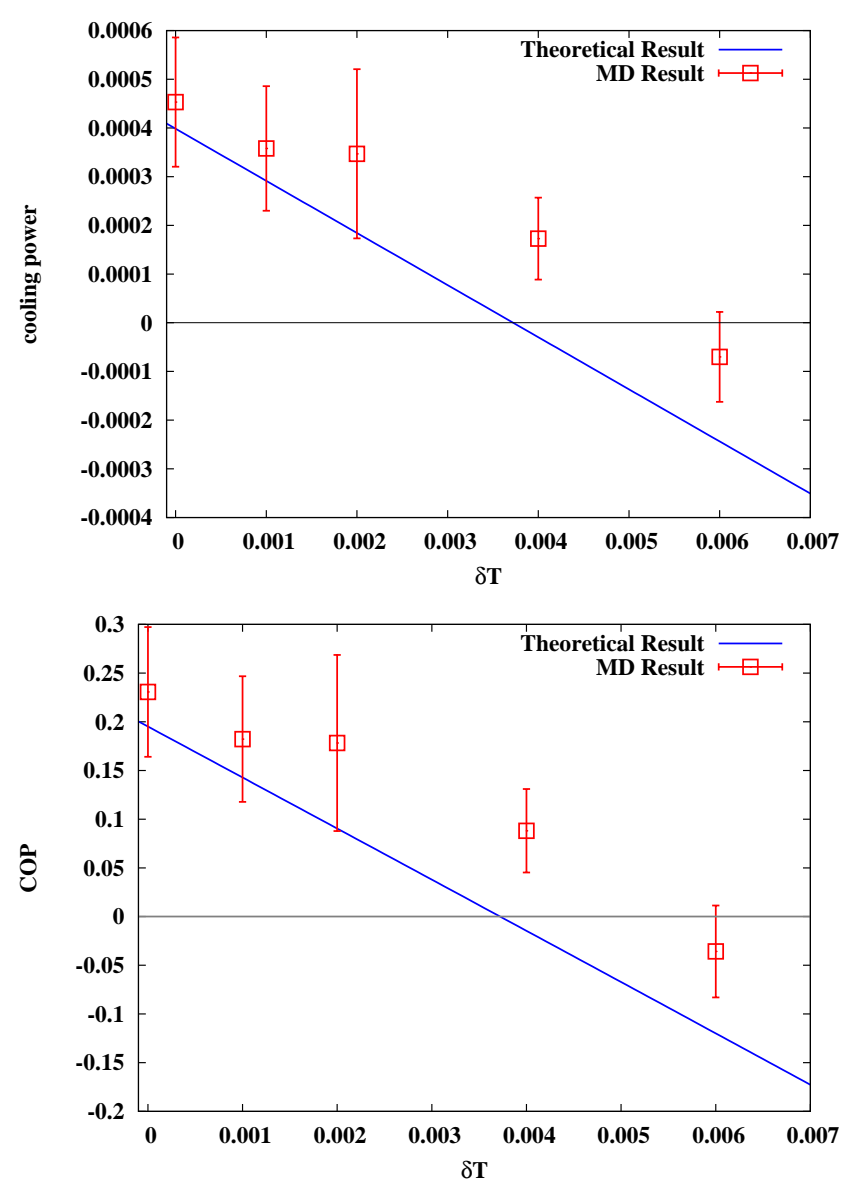

Fig. 10. The temperature difference $\delta T=T_{h}-T_{c}$ dependence of the cooling power and the COP, with the process durations $\Delta t_{\mathrm{sep}}=5000, \Delta t_{\mathrm{mix}}=3000$, and the temperatures $T_{h}=1+$ $\delta T / 2$ and $T_{c}=1-\delta T / 2$. The MD data were averaged over 6000 cycles.

Finally, we discuss some remaining problems. First, we can find that the COP is only about $0.2 \%$ of the Carnot $\mathrm{COP}$ at most from Figure 7 , but we have not yet found the conditions to obtain a heat pump model with much 
higher COP. To know the best performance of our model, we will need more thorough search on the parameter space of our model, though our search in the present study was limited to where our theoretical assumptions are probable. Second, it is difficult to realize our model experimentally since the Coulomb interaction between particles is ignored. To overcome this problems, our model should be generalized to consider the Coulomb interaction, for example by using MHD [27, 28, 29, 30]. We consider that experiments of our model become more realizable by removing the electric charges of particles and replacing the electric force with the gravity or inertial force such as centrifugal force $31,32,33$. In this replacement, the components of a gas mixture can be separated by the pressure gradient created by the gravity or the centrifugal force 2 . If a centrifuge is used, the separating process and the mixing process can be caused by accelerating and decelerating the angular velocity of the centrifuge, respectively, when different masses are given to the components of the gas mixture. Though our model in this paper may be merely a toy model, we expect that our work will trigger more realistic applications of the Dufour effect.

The authors would like to thank K. Nemoto, T. Nogawa, Y. Tami, and Y. Izumida for fruitful discussions.

\section{Author contribution statement}

M.H. mainly contributed to all the contents of this study including the preparation of the manuscript. K.O. supervised this study and is also responsible for all the contents.

\section{A Two dimensional expressions for the thermal-diffusion ratio $k_{T}$ and the coefficient $\lambda$}

The three-dimensional microscopic expression for $k_{T}$ of a binary mixture is obtained in reference [14 by approximately solving the subdivided Boltzmann equations by the Enskog method (see Sect. 8 in Ref. 14]). From the similar derivation to the three-dimensional expression, the two-dimensional expression in the first-order approximation denoted by $\left[k_{T}\right]_{1}$ is proved to be written as

$$
\begin{array}{r}
{\left[k_{T}\right]_{1}=2\left\{x_{A} M_{A}^{-\frac{1}{2}}\left(a_{-1-1} a_{01}-a_{0-1} a_{1-1}\right)\right.} \\
\left.+x_{B} M_{B}^{-\frac{1}{2}}\left(a_{0-1} a_{11}-a_{01} a_{1-1}\right)\right\} \\
\quad /\left(a_{-1-1} a_{11}-a_{1-1}^{2}\right)
\end{array}
$$

where $M_{A} \equiv m_{A} /\left(m_{A}+m_{B}\right), M_{B} \equiv m_{B} /\left(m_{A}+m_{B}\right)$, and the matrix elements $a_{11}, a_{1-1}, a_{-1-1}, a_{01}$ and $a_{0-1}$

\footnotetext{
${ }^{2}$ This mechanism of the separation is sometimes called the
} barodiffusion effect 34,35. in equation (A.1) are given by

$$
\begin{aligned}
& a_{11}=x_{A}^{2} \hat{\Omega}_{1}^{(2)}(2)+2 x_{A} x_{B}\left\{\left(6 M_{A}^{2} M_{B}+4 M_{B}^{3}\right) \hat{\Omega}_{12}^{(1)}(1)\right. \\
& -4 M_{B}^{3} \hat{\Omega}_{12}^{(1)}(2)+M_{B}^{3} \hat{\Omega}_{12}^{(1)}(3) \\
& \left.+2 M_{A} M_{B}^{2} \hat{\Omega}_{12}^{(2)}(2)\right\} \text {, } \\
& a_{-1-1}=2 x_{A} x_{B}\left\{\left(6 M_{B}^{2} M_{A}+4 M_{A}^{3}\right) \hat{\Omega}_{12}^{(1)}(1)\right. \\
& -4 M_{A}^{3} \hat{\Omega}_{12}^{(1)}(2)+M_{A}^{3} \hat{\Omega}_{12}^{(1)}(3) \\
& \left.+2 M_{B} M_{A}^{2} \hat{\Omega}_{12}^{(2)}(2)\right\}+x_{B}^{2} \hat{\Omega}_{2}^{(2)}(2), \\
& a_{1-1}=2 x_{A} x_{B} M_{A}^{\frac{3}{2}} M_{B}^{\frac{3}{2}}\left\{-\hat{\Omega}_{12}^{(1)}(3)+4 \hat{\Omega}_{12}^{(1)}(2)\right. \\
& \left.-10 \hat{\Omega}_{12}^{(1)}(1)+2 \hat{\Omega}_{12}^{(2)}(2)\right\}, \\
& a_{01}=2 x_{A} x_{B} M_{A}^{\frac{1}{2}}\left(2 M_{B}^{2} \hat{\Omega}_{12}^{(1)}(1)-M_{B}^{2} \hat{\Omega}_{12}^{(1)}(2)\right), \\
& a_{0-1}=-x_{A} x_{B} 2 M_{B}^{\frac{1}{2}}\left(2 M_{A}^{2} \hat{\Omega}_{12}^{(1)}(1)-M_{A}^{2} \hat{\Omega}_{12}^{(1)}(2)\right) \text {, }
\end{aligned}
$$

respectively. Here, $\hat{\Omega}_{12}^{(l)}(r), \hat{\Omega}_{1}^{(l)}(r)$ and $\hat{\Omega}_{2}^{(l)}(r)(l, r=1,2, \cdots)$ are defined as

$$
\begin{aligned}
& \hat{\Omega}_{12}^{(l)}(r)=\frac{1}{2} \sigma\left(\frac{2 k_{B} T}{m_{0} M_{A} M_{B}}\right)^{\frac{1}{2}} \hat{\mathcal{W}}^{(l)}(r), \\
& \hat{\Omega}_{1}^{(l)}(r)=\frac{1}{2} \sigma\left(\frac{k_{B} T}{m_{A}}\right)^{\frac{1}{2}} \hat{\mathcal{W}}^{(l)}(r), \\
& \hat{\Omega}_{2}^{(l)}(r)=\frac{1}{2} \sigma\left(\frac{k_{B} T}{m_{B}}\right)^{\frac{1}{2}} \hat{\mathcal{W}}^{(l)}(r),
\end{aligned}
$$

respectively, where $m_{0} \equiv m_{A}+m_{B}, \sigma$ is the diameter of the particles, and $\hat{\mathcal{W}}^{(l)}(r)$ are the non-dimensional values defined as

$$
\hat{\mathcal{W}}^{(l)}(r) \equiv 2 \int_{0}^{\infty}\left\{\int_{0}^{1} e^{-g^{2}} g^{2 r+1}\left(1-\cos ^{l} \chi\right) d\left(\frac{b}{\sigma}\right)\right\} d\left(g^{2}\right) .
$$

The parameter $\chi$ in equation A.10 is the scattering angle between the particles with interaction potential $U^{\text {int }}(r)$ and is a function of the scattering parameters $g$ and $b$ written as

$$
\chi(g, b)=\pi-2 \int_{R}^{\infty}\left\{\frac{r^{4}}{b^{2}}\left(1-\frac{U^{\mathrm{int}}(r)}{k_{B} T g^{2}}\right)-r^{2}\right\}^{-\frac{1}{2}} d r
$$

where $R$ is the solution to

$$
1-\frac{U^{\mathrm{int}}(R)}{k_{B} T g^{2}}-\frac{b^{2}}{R^{2}}=0 .
$$

Using the Herzian potential in equation (3) as $U^{\text {int }}(r)$ above, we can finally obtain equation (50) as the first order approximation of $k_{T}$. 
In the same way, the two-dimensional expression for $\lambda$ in the first-order approximation denoted by $[\lambda]_{1}$ can be obtained as

$$
\begin{aligned}
{[\lambda]_{1}=4 k_{B}^{2} T\{} & x_{A}^{2} m_{A}^{-1} a_{-1-1}-2 x_{A} x_{B}\left(m_{A} m_{B}\right)^{-\frac{1}{2}} a_{1-1} \\
& \left.+x_{B}^{2} m_{B}^{-1} a_{11}\right\} /\left(a_{-1-1} a_{11}-a_{1-1}^{2}\right)
\end{aligned}
$$

using the similar derivation to the three-dimensional expression in reference [14.

\section{B Derivation of equation (26)}

To derive equation (26), we calculate the work $W$ done to the system during one cycle consisting of the separating and mixing processes, which is written as

$$
W=\sum_{i=1}^{N} \int d \boldsymbol{r}_{i} \cdot\left\{q_{i} \boldsymbol{E}+\sum_{j(\neq i)=1}^{N}\left(-\nabla_{i} U_{i j}^{\mathrm{int}}\right)+\boldsymbol{F}_{i}^{\mathrm{bath}}\right\}
$$

where $U_{i j}^{\text {int }} \equiv U^{\text {int }}\left(\left|\boldsymbol{r}_{i}-\boldsymbol{r}_{j}\right|\right)$ is the interaction potential (specifically Eq. (3)) between the $i$ th and $j$ th particles, $\boldsymbol{F}_{i}^{\text {bath }}$ is the force on the $i$ th particle from the heat baths, and the integral $\int d \boldsymbol{r}_{i}$ is evaluated along the trajectory of the $i$ th particle for one cycle of the system. From the first term of equation (B.1), we obtain

$$
\sum_{i} \int d \boldsymbol{r}_{i} \cdot q_{i} \boldsymbol{E}=\psi_{E}^{\text {initial }}-\psi_{E}^{\mathrm{final}}
$$

where $\psi_{E}$ is defined below equation (26) and we note that the mixing process does not contribute the above equation because the electric field vanishes. The second term of equation (B.1) can be written as

$$
\sum_{i} \int d \boldsymbol{r}_{i} \cdot \sum_{j(\neq i)=1}^{N}\left(-\nabla_{i} U_{i j}^{\mathrm{int}}\right)=-\int d U^{\mathrm{int}}
$$

where we defined $U^{\text {int }}$ as

$$
U^{\mathrm{int}} \equiv \frac{1}{2} \sum_{i=1}^{N} \sum_{j(\neq i)=1}^{N} U_{i j}^{\mathrm{int}}
$$

Since the integral is evaluated for one cycle, equation (B.3) represents a change of the total interparticle potential between the beginning and the ending of a cycle. Therefore the second term of equation (B.1) should macroscopically be zero as long as the system is cyclic. Finally, the third term of equation (B.1) can be written as

$$
\sum_{i} \int d \boldsymbol{r}_{i} \cdot \boldsymbol{F}_{i}^{\mathrm{bath}}=-Q_{h}+Q_{c}
$$

from the definitions of $Q_{h}$ and $Q_{c}$.
Using equations (B.2), (B.3) and (B.5), we obtain

$$
W=\psi_{E}^{\text {initial }}-\psi_{E}^{\text {final }}-Q_{h}+Q_{c} .
$$

Because the denominator of the COP should be the work done by the external field except for the heat baths, $W_{E}$ in equation (25) can be written as equation (26).

\section{References}

1. L. Dufour, Ann. Phys. 148, 490 (1873).

2. P. M. Peltier, Annal. Chim. Phys. 56, 371 (1834).

3. D. M. Rowe, Thermoelectrics handbook, Macro to Nano (Taylor \& Frances, 2006)

4. H. J. Goldsmid, Applications of thermoelectricity (John Wiley, 1960)

5. L. Miller, Z. Naturforsch 4a, 262 (1949).

6. R. L. Rowley, F. H. Horne, J. Chem. Phys. 68, 325 (1978).

7. R. L. Rowley, F. H. Horne, J. Chem. Phys. 72, 131 (1980).

8. L. Waldmann, Z. Phys. 124, 2 (1947).

9. L. Waldmann, Z. Naturforsch 4a, 195 (1949).

10. M. A. Korzhuev, Phys. Solid State 40, 242 (1998).

11. K. E. Grew, L. Thomas, Thermal Diffusion in Gases (Cambridge University Press, 1952)

12. S. R. De Groot, P. Mazur, Non-Equilibrium Thermodynamics (Dover Publications, 1984)

13. S. E. Ingle, F. H. Horne, J. Chem. Phys. 59, 5882 (1973).

14. S. Chapman, T. G. Cowling, The mathmatical theory of non-uniform gases, 3rd edn. (Cambridge University Press, 1970)

15. S. Chapman, Proc. R. Soc. A 177, 38 (1940).

16. L. Waldmann, Z. Phys. 121, 501 (1943).

17. R. F. Streater, Proc. R. Soc. A 456, 205 (2000).

18. R. G. Mortimer, H. Eyring, Proc. Natl. Acad. Sci. USA 77, 1728 (1980).

19. M. P. Allen, D. J. Tildesley, Computer simulation of liquids (Oxford Science Publications, 1987)

20. A. E. Love, in A Treatise on the Mathematical Theory of Elasticity, 4th edn. (Cambridge University Press, 1952) Chap. VIII-139

21. S. Yukawa, J. Phys. Soc. Jpn. 78, 0230002 (2009).

22. Y. Yuge, N. Ito, A. Shimizu, J. Phys. Soc. Jpn. 74, 1895 (2005).

23. R. Tehver, F. Toigo, J. Koplik, J. R. Banavar, Phys. Rev. E 57, R17 (1998).

24. J. R. Henderson, F. V. Swol, Mol. Phys. 51, 991 (1984).

25. I. K. Snook, D. Henderson, J. Chem. Phys. 68, 2134 (1978).

26. I. Prigogine, in Introduction to Thermodynamics of irreversible processes, 3rd edn. (Interscience, 1967) Chap. IV-2

27. R. Moreau, Magnetohydrodynamics (Kluwer Academic Publishers, 1990)

28. T. Hayat, S. A. Shehzad, A. Alsaedi, Appl. Math. Mech. 33, 1301 (2012).

29. A. J. Chamkha, A. M. Rashad, Canad. J. Chem. Eng. 92, 758 (2014).

30. M. Nawaz, T. Hayat, A. Alsaedi, Appl. Math. Mech. 33, 1403 (2012).

31. S. Whitley, Rev. Mod. Phys. 56, 41 (1984).

32. K. Cohen, The theory of isotope separation as applied to the largescale production of U235 (McGraw-Hill, 1951)

33. R. S. Kemp, Science and Global Security, 17, 1 (2009). 
34. B. R. Sharma, R. N. Singh, Heat Mass Transfer, 7, 769 (2010).

35. L. D. Landau, E. M. Lifshitz, Fluid Mechanics, 2nd edn. (Pergamon Press, 1987) 